\title{
Old Does Not Necessarily Mean Worse: Standard Stupp Regimen for Elderly Patients ( $\geq 70$ years) with Newly Diagnosed Glioblastoma
}

Loig Vaugier ( $D$ loig.vaugier@ico.unicancer.fr)

ICO Site René Gauducheau: Institut de Cancerologie de l'Ouest https://orcid.org/0000-0003-0728-0693

Loïc Ah-Thiane

ICO Site René Gauducheau: Institut de Cancerologie de l'Ouest

Maud Aumont

ICO Site René Gauducheau: Institut de Cancerologie de l'Ouest

\section{Emmanuel Jouglar}

ICO Site René Gauducheau: Institut de Cancerologie de l'Ouest

\section{Mario Campone}

ICO Site René Gauducheau: Institut de Cancerologie de l'Ouest

Camille Colliard

ICO Site René Gauducheau: Institut de Cancerologie de l'Ouest

\section{Ludovic Doucet}

ICO Site René Gauducheau: Institut de Cancerologie de l'Ouest

Jean-Sébastien Frenel

ICO Site René Gauducheau: Institut de Cancerologie de l'Ouest

Carole Gourmelon

ICO Site René Gauducheau: Institut de Cancerologie de l'Ouest

Marie Robert

ICO Site René Gauducheau: Institut de Cancerologie de l'Ouest

\section{Stéphane-André Martin}

CHU Nantes: Centre Hospitalier Universitaire de Nantes

\section{Tanguy Riem}

CHU Nantes: Centre Hospitalier Universitaire de Nantes

\section{Vincent Roualdes}

CHU Nantes: Centre Hospitalier Universitaire de Nantes

\section{Loïc Campion}

ICO Site René Gauducheau: Institut de Cancerologie de l'Ouest

\section{Augustin Mervoyer}

ICO Site René Gauducheau: Institut de Cancerologie de l'Ouest 


\section{Research Article}

Keywords: glioblastoma, elderly, radiotherapy, temozolomide, Stupp

Posted Date: February 24th, 2021

DOl: https://doi.org/10.21203/rs.3.rs-233647/v1

License: (c) (i) This work is licensed under a Creative Commons Attribution 4.0 International License. Read Full License 


\section{Abstract}

Introduction Glioblastoma (GBM) is frequent in elderly patients, but their frailty provokes debate regarding optimal treatment in general, and the standard Stupp regimen in particular, although this is the mainstay for younger ( $<65$ years) patients. Methods All patients with newly diagnosed GBM and age $\geq 70$ who were referred to our institution for Stupp treatment were reviewed from 2004 to 2018. MGMT status was not available for treatment decision. The primary endpoint was overall survival (OS). Secondary outcomes were relapse-free survival (RFS), early ( $\leq 1$ month after RCT) adverse neurological events (symptoms of intracranial hypertension and/or use of corticosteroids and/or hospitalization) and temozolomide hematologic toxicity assessed by CTCAE v5. Results 128 patients were included. The median age was 74.1 (IQR: $72-77$ ). $15 \%$ of patients were $\geq 80$ years. $62.5 \%$ and $37.5 \%$ of patients fulfilled the criteria for RPA class I-II and III-IV, respectively. $81 \%$ of patients received the entire RCT and $28 \%$ completed the maintenance temozolomide. With median follow-up of 11.7 months (IQR: 6.5-17.5), median OS was 11.7 months (Cl95\%: 10-13 months). Median RFS was 9.5 months (Cl95\%: 9-10.5 months). $60 \%$ of patients had early adverse neurological events, of whom $44 \%$ had progression and $8 \%$ experienced grade $\geq 3$ hematologic adverse events. RPA class III-IV and occurrence of neurological events were associated with lower OS rates, whereas post-operative neurological disabilities were not. Age $\geq 80$ was not associated with worsened outcomes. Conclusions Stupp radiochemotherapy was feasible and effective for "real-life" elderly patients diagnosed with glioblastoma, even in the case of post-operative neurological disabilities.

\section{Introduction}

Glioblastoma (GBM) is the most malignant and common primary brain tumor in adults [1]. The overall prognosis remains poor: around 12-15 months [2] [3]. The current therapeutics rely on surgical resection, radiotherapy (RT), chemotherapy (CT) and best supportive cares (BSC) [4]. The combination of 6 weeks of conventionally fractionated RT (CFRT) with radiosensitizing temozolomide (TMZ), followed by up to 6 cycles of maintenance TMZ (known as the standard Stupp regimen [3]) is the mainstay for $<65$ year-old patients [4]. However, there are concerns about whether and/or which elderly patients may benefit from such post-operative treatment.

Focusing on the elderly population (e.g. aged $\geq 70$ years) with newly-diagnosed GBM is relevant for the following reasons: i) the highest incidence rate is currently observed in patients aged 75 to 84 years [5] ; ii) neurological symptoms (following progression or treatment toxicities) may have dramatic consequences on independence and/or quality of life for such a frail population [6] [7]; iii) the life expectancy is extremely poor but has increased in the last decade with the development of post-operative treatments [2]; iv) GBM-specific geriatric scales of frailty are still lacking. Age and performance status (PS) >2 are common negative prognostic factors [8] [9]. MGMT (06-methylguanine-DNA methyltransferase) DNA-repair gene silencing and its consequence on therapeutics have been investigated considerably [10] [11]. In particular, MGMT methylation is associated with improved 
response to TMZ. The difficulties in interpreting the results of the tests nevertheless mean that MGMT status not routinely assessed [12].

Over the last few years, both the indication for, and modalities of, post-operative treatments in elderly patients have been controversial [13] [14] [15] [16]. Patients $>70$ years were not included in the original study by Stupp et al. [3]. 65-70 year-old patients may benefit from the Stupp regimen compared to RT alone, particularly in case of PS $\leq 1$ and macroscopically complete surgical resection [17] [18] [19] [20] [21] [22] [23] [24] [25] [26]. For patients $>70$ years, post-operative RT could result in only modest improvements compared to BSC and despite a Karnofsky index $>70$, whereas the duration of the CFRT may represent almost one third of their life expectancy [27]. Hypofractionated and accelerated RT protocols over 1 or 3 weeks (HFRT) have emerged in this context, with outcomes comparable with those of CFRT and acceptable tolerance [28] [29] [30] [31] [32]. Recently, a large phase 3 trial has shown the survival benefits of HFRT with TMZ versus HFRT alone for $>65$ year-old and PS $\leq 2$ patients [33]. This type of regimen tends to be the current standard of care for elderly patients although there are no prospective trials comparing it to the standard Stupp protocol. Interestingly, two ongoing phase 3 trials: EORTC-1709-BTG (NCT03345095) (RT+TMZ and marizomib); and RT "dose painting" escalation + TMZ (SPECTRO-GLIO, NCT01507506) [34], have contributed to bringing to the fore the standard Stupp regimen for patients with no upper limit of age.

In this context, we present the tolerance data and outcomes for all the elderly patients ( $\geq 70$ years) who were referred to our institution for the standard Stupp regimen. The objective was to investigate whether common geriatric sources of frailty such as age or baseline neurological disabilities, had a negative impact on survival.

\section{Materials And Methods}

\section{Patient selection}

All $\geq 70$-year-old, histologically-proven GBM patients referred to our radiation therapy department (Institut de Cancérologie de l'Ouest, Saint Herblain, France) for a Stupp regimen from January 2004 to December 2018 were included. Patients with World Health Organisation (WHO) grade <4 gliomas were excluded [1]. All patients had surgical intervention - either complete (CR) or partial (PR) resection or biopsy (BR) - and were considered fit for post-operative Stupp radiochemotherapy (RCT) by a multidisciplinary team including neurosurgeons, and medical and radiation oncologists. The histomolecular isocitrate deshydrogenase (IDH) mutation was determined in each case. MGMT-methylation status was not considered informative for therapeutic decisions and was not carried out routinely.

\section{Post-operative treatment modalities and follow-up}

Immobilization in the treatment position was systematically achieved using custom thermoplastik mask contention during RT. An RT-dedicated computed tomography (CT) scan was registered with contrastenhanced T1-weighted brain magnetic resonance imaging (MRI) in order to guide tumor 
delineation [35] [36]. The gross tumor volume (GTV) was defined as the contrast enhancement area in the T1-weighted MRI sequence and CT scan, including the tumor bed for patients with prior partial or complete resection. Following GBM guidelines [36], the clinical target volume (CTV) was defined as the addition of a geometric tridimensional $10-20 \mathrm{~mm}$ margin (depending on the tumor's topography) around the GTV that was corrected to the anatomical borders and had to include the hypersignal FLAIR-MRI around the GTV. The planning target volume (PTV) was defined as CTV $+5 \mathrm{~mm}$. The dose prescribed to the PTV was 60 Gy in 30 fractions of $2 \mathrm{~Gy}$ per fraction, 5 days a week within conformal three-dimensional radiotherapy [3]. Concomitant daily TMZ ( $75 \mathrm{mg} / \mathrm{m}^{2}, 7$ days a week from the first to the last day of RT) was prescribed during RT, with weekly blood samples.

All patients were examined by their medical oncologists one month after the last RT session to start up to 6 cycles of maintenance TMZ (150-200mg/m², 5 consecutive days a month). During the RCT, treatment tolerance was evaluated once a week. Patients were followed up clinically and with blood tests once a month throughout the maintenance phase and then every three months. The first brain MRI for evaluation was performed three months after the end of RT, then every three months for at least five years.

\section{Outcomes}

The primary endpoint was overall survival (OS). Secondary endpoints were relapse-free survival (RFS), incidence rate for early adverse neurological events, and TMZ-related toxicity assessed by the CTCAE v5 classification.

Survivals (OS and RFS) were respectively defined as the time from histological diagnosis to death from any cause, and disease recurrence as assessed by MRI (at least T1 with gadolinium injection and FLAIR). As it can be difficult to distinguish recurrence from pseudoprogression after RT and TMZ [37], repeated MRI over shorter time interval than planned were necessary in some cases. The date of progression assigned was the earlier date when progression was first suspected. Early ( $\leq 1$ month after RCT) adverse neurological events were defined as the occurrence of symptoms of intracranial hypertension (ICHT) and/or the use of corticosteroids and/or the need for hospitalization for any cause: during RCT (RCTconcomitant); and between the end of RCT and the start of maintenance TMZ (post-RCT).

For each patient, the presence of neurological disabilities (motor, visual, instability, cognitive and communication) was also reviewed before and after RCT. Motor disabilities were scored between mild (e.g. paresis) and severe (e.g. objective neurological deficit). Visual disabilities corresponded to homonymous hemianopias or anopsias. Instability included dizziness and proprioceptive disorders; cognitive disabilities included disorientation in time and place, frontal syndrome or mnesic disorders.

\section{Statistical analysis}

Qualitative factors were described in terms of the frequency of their respective modalities and compared using of Pearson's Chi-square test (or Fisher test). For continuous factors, independent groups were described by means of their median [range] and compared using a Student's t-test (or Mann-Whitney). 
Survival (RFS and OS) was described by means of Kaplan-Meier curves and compared between interest groups using log-rank tests. Median follow-up was calculated by means of inverse Kaplan-Meier method. Univariate logistic regressions were performed to assess prognostic factors on the occurrence of adverse neurological events. The RPA class by Scott et al. [8] - I (II): $\leq(>) 75.5$ with CR/PR; III (IV): PS $\leq(>) 1$ with BR - was considered. All tests were two-sided; significance was set at $p=0.05$, all calculations were made using Stata 16.1 SE (StataCorp LLC, College Station, Texas, USA).

This study was approved by the ethic committee of the Centre Hospitalo-Universitaire (CHU) in Angers, France (Number 2020/117).

\section{Results}

\section{Patient and treatment characteristics}

A cohort of 128 patients was established from January 2004 to December 2018. Patient characteristics are summarized in Table 1. All patients had IDH wild-type GBM. The median age was 74.1 (IQR: 72-77) with 73/128 (57\%) male. Most patients had PS 0 (49/128; 38.5\%) and 1 (71/128; 55.5\%). $39 \%, 23.5 \%$, $33.5 \%$ and $4 \%$ patients respectively fulfilled the criteria for RPA class I, II, III and IV. 19/128 (15\%) were $\geq 80$ years old, with 11 RPA II, 5 RPA III and 3 RPA IV.

104 patients (81\%) received the entire Stupp RCT and 36 (28\%) completed the further 6 maintenance TMZ cycles. $13(10 \%)$ patients did not fulfill the 30 RT fractions because of major overall worsening or death; 11 (9\%) patients received 60 Gy but $<6$ concomitant TMZ cycles because of blood toxicity or swallowing troubles for one patient. The median number for RT fractions, concomitant TMZ weeks and maintenance TMZ months were respectively 30 (IQR, 30-30), 6 (IQR, 6-6) and 2 (IQR, 0-6). All $\geq 80$-year-old patients received the $30 \mathrm{RT}$ fractions.

Of the 84 patients with progression, $51 / 84(60.7 \%)$ and $33 / 84(39.3 \%)$ patients had respectively a second line of chemotherapy and BSC following progression.

\section{Overall and progression-free survivals}

Oncologic outcomes are summarized in Table 2 and Figure 1. With a median follow-up of 11.7 months (IQR: 6.5-17.5), the median OS was 11.7 months (CI95\%: 10-13 months). The 2- and 5-year OS was 15\% (CI95\%: 10-22\%) and 2.4\% (CI95\%: 0.6-6\%), respectively. The median RFS was 9.5 months (CI95\%: 9-10.5 months).

$60 \%(77 / 128)$ of patients had either RCT-concomitant $(39 \%)$ or post-RCT $(45.5 \%)$ adverse neurological events, of whom $44 \%$ (34/77) had progression. The rate for grade $\geq 3 \mathrm{TMZ}$-induced blood toxicity (mainly thrombopenia) yielded $8 \%(10 / 128) .58 .5 \%(75 / 128)$ and $57 \%(73 / 128)$ had pre- and post-RCT neurological disabilities, of whom $4.5 \%(6 / 128)$ were severe prior to the RCT. One patient had fully regressive facial paralysis after treatment, while the neurological symptoms were stable for the other five. 
Regarding the $\geq 80$-year-old cohort, the median OS and RFS were 12.1 and 9.2 months, respectively. The rate for RCT-concomitant and post-RCT adverse neurological events was $16.5 \%$ and $44.5 \%$, respectively.

\section{Prognostic factors for death, post-treatment neurological events, and treatment completion}

Prognostic factors for death from any cause and post-RCT adverse neurological events are summarized in Figure 3. The quality of the surgical resection (CR-PR versus BR; HR =0.62, $p=0.011$ ), RPA class (I-II versus III-IV; HR = 0.61, $p=0.008)$, post-RCT neurological disabilities ( $H R=1.54, p=0.017)$, RCTconcomitant and post-RCT adverse neurological events $(H R=1.91, p<0.001$ and $H R=2.67, p<0.001$, respectively) were significantly associated with death from any cause in univariate analysis but this was not the case for either age $\geq 80(H R=1.03, p=0.90)$ or pre-RCT neurological disabilities $(H R=1.01, p=$ 0.08) (Figure 2).

Patients with pre-RCT neurological disabilities did not exhibit significantly higher occurrence for post-RCT adverse neurological events $(O R=0.88, p=0.072)$ (Figure 3$)$, nor did $\geq 80$-year-old patients $(O R=1.10, p$ $=0.84)$. The quality of the surgical resection (CR-PR versus BR; OR $=0.46, p=0.037$ ) and RPA class (I-II versus III-IV, OR $=0.43, p=0.023$ ) were significantly associated with lower incidence for such events.

Neither age $\geq 80(p=0.21)$, BR $(p=0.22)$, III-IV RPA class $(p=0.19)$ nor pre-RCT neurological disabilities $(p=0.86)$, were associated with incomplete RCT.

\section{Discussion}

Around $80 \%$ of all the $\geq 70$-year-old patients who were referred to our institution for post-operative Stupp treatment, received the entire 6-week long Stupp RCT. In particular, all the $\geq 80$-year-old patients completed this treatment. The RPA classification by Scott et al. [8] was prognostic for both the OS and occurrence of early adverse neurological events, but interestingly, the presence of neurologic disabilities at baseline was not associated with worsened outcomes.

In recent decades, several studies have investigated different treatment modalities to go further than the Stupp protocol: e.g. CT intensification with lomustine for MGMT-methylated patients [38], TMZ dose escalation [39] or the addition of irinotecan during the maintenance TMZ phase [40]; maintenance TMZ beyond 6 cycles [41]; the addition of antiangiogenic drugs such as bevacizumab [42] [43] or cilengitide [44]; immunotherapeutic approaches with vaccines such as Rindopepimut ${ }^{\circledR}$ for patients with a mutation in the epidermal growth factor receptor (EGFR) gene [45] or antiPD1 checkpoint inhibitors [46]; alternating electric fields to the brain called Tumor Treating Fields (TTF) during the maintenance phase [47]. At this time, none of these treatments except TTF has been able to demonstrate clear oncologic improvements compared to the original Stupp regimen, which remains the mainstay for $\leq 65-$ 70-year-old patients with median RFS and OS of 6.9 and 14.6 months, respectively [3]. In comparison, median OS were 3.9-9.6 months with exclusive RT or TMZ [28] [30] [33] [48]. Whereas age commonly acts as an obstacle for the standard Stupp treatment, the results of our study show that i) standard RCT (>80\% completion rate) was feasible for $\geq 70$ and even $\geq 80$-year-old GBM patients; ii) the survival rates 
(median OS of 12 months regardless of MGMT status) were rather comparable to the values for trialselected and/or younger patients. Similar prospective/retrospective analyses have already corroborated this observation [18] [19] [20] [21] [22] [23] [24] [25] [26].

As the oncologic outcomes unfortunately remain poor in GBM, tolerance and consequences on quality of life are crucial, especially for the elderly population. Around $60 \%$ of patients were concerned by posttreatment adverse neurological events, while around half of them had no visible progression. The occurrence of such events were correlated with lower OS as already described in the literature [9]. The presence of neurological disability at baseline was associated with neither worsened OS nor higher occurrence of post-treatment neurological events. This may reflect the quality of the surgical resection, which had a further, positive impact on survival. Although often considered as a source of geriatric frailty, the presence of neurological disability alone should not be a reason for post-operative de-escalation.

Lastly, the treatment modalities and duration for the Stupp CFRT may respectively seem too heavy and too long compared to the life expectancy of the patients - although it was generally similar in our study with younger patients. Only $28 \%$ of the cohort completed the entire regimen with the maintenance TMZ. Various therapeutic options specifically aimed at the elderly have emerged in this regard. Accelerated HFRT +/- TMZ is increasingly being used with significantly lower radiation doses but paradoxically comparable outcomes [28] [29] [30] [31] [32] [33]. The overall good tolerance and brain diffusion also make TMZ monotherapy an option [28] [48] [49], especially in the case of MGMT methylation [10] [11] [12]. The fear of therapeutic de-escalation arising from such protocols means they are not recommended for younger patients [26]. Some elderly patients could however appear suitable for the best and maybe most aggressive strategy, but reliable predictive biomarkers are lacking. The development of GBM-dedicated geriatric scales e.g. relying on the RPA classification, appears crucial for optimizing treatment algorithms [13] [14].

Our study has obvious limitations, mainly linked to its retrospective nature. Nutrition and mood assessments are other important geriatric parameters, but the data were incomplete or missing from our recording. The patients included in this study were treated before the implementation of a systematic geriatric assessment in our department. Overall prospective geriatric evaluation is needed to build GBMdedicated treatment algorithms.

\section{Conclusions}

Standard Stupp radiochemotherapy was feasible for "real-life" elderly patients diagnosed with glioblastoma with unknown MGMT status, even in cases of post-operative neurological disabilities. GBMdedicated geriatric scales are urgently needed to guide optimal therapeutics.

\section{Declarations}

Funding: Not applicable. 
Conflict of interest: The authors declare that they have no known competing financial interests or personal relationships that could have appeared to influence the work reported in this paper.

Availability of data and material: Research data are stored in an institutional repository and will be shared upon request to the corresponding author.

\section{Authors' contributions:}

Conceptualization, L.V. and A.M.; methodology, L.V., L.A-T. and L.C.; software, L.C.; validation, L.V., L.A-T., L.C. and A.M.; formal analysis, L.V., L.A-T. and L.C.; investigation, L.V. and L.A-T.; resources, L.V. and L.A-T.; data curation, L.V., L.A.T. and L.C.; writing-original draft preparation, L.V., L.A-T., L.C. and A.M.; writingreview and editing, all authors; visualization, L.V. and L.C.; supervision, A.M.; project administration, A.M.; All authors have read and agreed to the published version of the manuscript.

Ethics approval: This study was approved by the ethic committee of the Centre Hospitalo-Universitaire (CHU) in Angers, France (Number 2020/117).

\section{Bibliography}

[1] D. Ricard, A. Idbaih, F. Ducray, M. Lahutte, K. Hoang-Xuan, and J.-Y. Delattre, "Primary brain tumours in adults," Lancet, vol. 379, no. 9830, pp. 1984-1996, May 2012, doi: 10.1016/S01406736(11)61346-9.

[2] B. K. Shah, A. Bista, and S. Sharma, "Survival Trends in Elderly Patients with Glioblastoma in the United States: a Population-based Study," Anticancer Res., vol. 36, no. 9, pp. 4883-4886, 2016, doi: 10.21873/anticanres.11052.

[3] R. Stupp et al., "Radiotherapy plus concomitant and adjuvant temozolomide for glioblastoma," $N$. Engl. J. Med., vol. 352, no. 10, pp. 987-996, Mar. 2005, doi: 10.1056/NEJMoa043330.

[4] M. Weller et al., "European Association for Neuro-Oncology (EANO) guideline on the diagnosis and treatment of adult astrocytic and oligodendroglial gliomas," Lancet Oncol., vol. 18, no. 6, pp. e315e329, 2017, doi: 10.1016/S1470-2045(17)30194-8.

[5] Q. T. Ostrom, H. Gittleman, G. Truitt, A. Boscia, C. Kruchko, and J. S. Barnholtz-Sloan, "CBTRUS Statistical Report: Primary Brain and Other Central Nervous System Tumors Diagnosed in the United States in 2011-2015," Neuro-oncology, vol. 20, no. suppl_4, pp. iv1-iv86, 01 2018, doi: 10.1093/neuonc/noy131.

[6] C. F. Lorimer, F. Saran, A. J. Chalmers, and J. Brock, "Glioblastoma in the elderly - How do we choose who to treat?," J Geriatr Oncol, vol. 7, no. 6, pp. 453-456, 2016, doi: 10.1016/j.jgo.2016.07.005.

[7] S. Hoffe and L. Balducci, "Cancer and age: general considerations," Clin. Geriatr. Med., vol. 28, no. 1, pp. 1-18, Feb. 2012, doi: 10.1016/j.cger.2011.09.001. 
[8] J. G. Scott et al., "Recursive partitioning analysis of prognostic factors for glioblastoma patients aged 70 years or older," Cancer, vol. 118, no. 22, pp. 5595-5600, Nov. 2012, doi: 10.1002/cncr.27570.

[9] R. Rahman et al., "Incidence, risk factors, and reasons for hospitalization among glioblastoma patients receiving chemoradiation," J. Neurooncol., vol. 124, no. 1, pp. 137-146, Aug. 2015, doi: 10.1007/s11060-015-1820-3.

[10] M. E. Hegi et al., "MGMT gene silencing and benefit from temozolomide in glioblastoma," N. Engl. J. Med., vol. 352, no. 10, pp. 997-1003, Mar. 2005, doi: 10.1056/NEJMoa043331.

[11] E. R. Gerstner, S. Yip, D. L. Wang, D. N. Louis, A. J. lafrate, and T. T. Batchelor, "Mgmt methylation is a prognostic biomarker in elderly patients with newly diagnosed glioblastoma," Neurology, vol. 73, no. 18, pp. 1509-1510, Nov. 2009, doi: 10.1212/WNL.0b013e3181bf9907.

[12] M. E. Hegi et al., "MGMT Promoter Methylation Cutoff with Safety Margin for Selecting Glioblastoma Patients into Trials Omitting Temozolomide: A Pooled Analysis of Four Clinical Trials," Clin. Cancer Res., vol. 25, no. 6, pp. 1809-1816, 15 2019, doi: 10.1158/1078-0432.CCR-18-3181.

[13] J. T. Jordan, E. R. Gerstner, T. T. Batchelor, D. P. Cahill, and S. R. Plotkin, “Glioblastoma care in the elderly," Cancer, vol. 122, no. 2, pp. 189-197, Jan. 2016, doi: 10.1002/cncr.29742.

[14] A. Asmaa et al., "Management of elderly patients with glioblastoma-multiforme-a systematic review," Br J Radiol, vol. 91, no. 1088, p. 20170271, 2018, doi: 10.1259/bjr.20170271.

[15] G. Minniti, G. Lombardi, and S. Paolini, "Glioblastoma in Elderly Patients: Current Management and Future Perspectives," Cancers, vol. 11, no. 3, Mar. 2019, doi: 10.3390/cancers11030336.

[16] A. Fiorentino et al., "Can elderly patients with newly diagnosed glioblastoma be enrolled in radiochemotherapy trials?," Am. J. Clin. Oncol., vol. 38, no. 1, pp. 23-27, Feb. 2015, doi: 10.1097/COC.0b013e3182868ea2.

[17] R. Stupp et al., "Effects of radiotherapy with concomitant and adjuvant temozolomide versus radiotherapy alone on survival in glioblastoma in a randomised phase III study: 5-year analysis of the EORTC-NCIC trial," Lancet Oncol., vol. 10, no. 5, pp. 459-466, May 2009, doi: 10.1016/S14702045(09)70025-7.

[18] C. Gzell, H. Wheeler, L. Guo, M. Kastelan, and M. Back, "Elderly patients aged 65-75 years with glioblastoma multiforme may benefit from long course radiation therapy with temozolomide," J. Neurooncol., vol. 119, no. 1, pp. 187-196, Aug. 2014, doi: 10.1007/s11060-014-1472-8.

[19] E. Franceschi et al., "Which elderly newly diagnosed glioblastoma patients can benefit from radiotherapy and temozolomide? A PERNO prospective study," J. Neurooncol., vol. 128, no. 1, pp. 157162, 2016, doi: 10.1007/s11060-016-2093-1. 
[20] G. Minniti et al., "Radiotherapy plus concomitant and adjuvant temozolomide for glioblastoma in elderly patients," J Neurooncol, vol. 88, no. 1, pp. 97-103, May 2008, doi: 10.1007/s11060-008-9538-0.

[21] C. A. Barker et al., "Radiotherapy and concomitant temozolomide may improve survival of elderly patients with glioblastoma," J Neurooncol, vol. 109, no. 2, pp. 391-397, Sep. 2012, doi: 10.1007/s11060012-0906-4.

[22] J. Biau et al., "Radiotherapy plus temozolomide in elderly patients with glioblastoma: a 'real-life' report," Radiat Oncol, vol. 12, no. 1, p. 197, Dec. 2017, doi: 10.1186/s13014-017-0929-2.

[23] C. N. Chang-Halpenny, J. Yeh, and W. W. Lien, "Elderly patients with glioblastoma multiforme treated with concurrent temozolomide and standard- versus abbreviated-course radiotherapy," Perm J, vol. 19, no. 1, pp. 15-20, 2015, doi: 10.7812/TPP/14-083.

[24] S. E. Combs et al., "Postoperative Treatment of Primary Glioblastoma Multiforme With Radiation and Concomitant Temozolomide in Elderly Patients," International Journal of Radiation

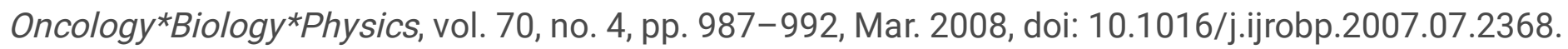

[25] J. Gerstein et al., "Postoperative radiotherapy and concomitant temozolomide for elderly patients with glioblastoma," Radiotherapy and Oncology, vol. 97, no. 3, pp. 382-386, Dec. 2010, doi: 10.1016/j.radonc.2010.06.014.

[26] C. Hanna et al., "Treatment of newly diagnosed glioblastoma in the elderly: a network metaanalysis," Cochrane Database Syst Rev, vol. 3, p. CD013261, Mar. 2020, doi:

10.1002/14651858.CD013261.pub2.

[27] F. Keime-Guibert et al., "Radiotherapy for glioblastoma in the elderly," N. Engl. J. Med., vol. 356, no. 15, pp. 1527-1535, Apr. 2007, doi: 10.1056/NEJMoa065901.

[28] A. Malmström et al., "Temozolomide versus standard 6-week radiotherapy versus hypofractionated radiotherapy in patients older than 60 years with glioblastoma: the Nordic randomised, phase 3 trial," Lancet Oncol., vol. 13, no. 9, pp. 916-926, Sep. 2012, doi: 10.1016/S1470-2045(12)702656.

[29] W. Roa et al., "International Atomic Energy Agency Randomized Phase III Study of Radiation Therapy in Elderly and/or Frail Patients With Newly Diagnosed Glioblastoma Multiforme," J. Clin. Oncol., vol. 33, no. 35, pp. 4145-4150, Dec. 2015, doi: 10.1200/JC0.2015.62.6606.

[30] W. Roa et al., "Abbreviated course of radiation therapy in older patients with glioblastoma multiforme: a prospective randomized clinical trial," J. Clin. Oncol., vol. 22, no. 9, pp. 1583-1588, May 2004, doi: 10.1200/JCO.2004.06.082.

[31] N. D. Arvold et al., "Hypofractionated versus standard radiation therapy with or without temozolomide for older glioblastoma patients," Int. J. Radiat. Oncol. Biol. Phys., vol. 92, no. 2, pp. 384- 
389, Jun. 2015, doi: 10.1016/j.jjrobp.2015.01.017.

[32] G. Minniti et al., "Standard (60 Gy) or short-course (40 Gy) irradiation plus concomitant and adjuvant temozolomide for elderly patients with glioblastoma: a propensity-matched analysis," Int. J. Radiat. Oncol. Biol. Phys., vol. 91, no. 1, pp. 109-115, Jan. 2015, doi: 10.1016/j.ijrobp.2014.09.013.

[33] J. R. Perry et al., "Short-Course Radiation plus Temozolomide in Elderly Patients with Glioblastoma," N. Engl. J. Med., vol. 376, no. 11, pp. 1027-1037, 16 2017, doi: 10.1056/NEJMoa1611977.

[34] A. Laprie et al., "Dose-painting multicenter phase III trial in newly diagnosed glioblastoma: the SPECTRO-GLIO trial comparing arm A standard radiochemotherapy to arm B radiochemotherapy with simultaneous integrated boost guided by MR spectroscopic imaging," BMC Cancer, vol. 19, no. 1, p. 167, Feb. 2019, doi: 10.1186/s12885-019-5317-x.

[35] E. P. Sulman et al., "Radiation Therapy for Glioblastoma: American Society of Clinical Oncology Clinical Practice Guideline Endorsement of the American Society for Radiation Oncology Guideline," J. Clin. Oncol., vol. 35, no. 3, pp. 361-369, Jan. 2017, doi: 10.1200/JC0.2016.70.7562.

[36] L. Feuvret, D. Antoni, J. Biau, G. Truc, G. Noël, and J.-J. Mazeron, "[Guidelines for the radiotherapy of gliomas]," Cancer Radiother, vol. 20 Suppl, pp. S69-79, Sep. 2016, doi: 10.1016/j.canrad.2016.07.008.

[37] N. Verma, M. C. Cowperthwaite, M. G. Burnett, and M. K. Markey, "Differentiating tumor recurrence from treatment necrosis: a review of neuro-oncologic imaging strategies," Neuro Oncol, vol. 15, no. 5, pp. 515-534, May 2013, doi: 10.1093/neuonc/nos307.

[38] U. Herrlinger et al., "Lomustine-temozolomide combination therapy versus standard temozolomide therapy in patients with newly diagnosed glioblastoma with methylated MGMT promoter (CeTeG/NOA-09): a randomised, open-label, phase 3 trial," Lancet, vol. 393, no. 10172, pp. 678-688, 16 2019, doi: 10.1016/S0140-6736(18)31791-4.

[39] M. R. Gilbert et al., "Dose-dense temozolomide for newly diagnosed glioblastoma: a randomized phase III clinical trial," J. Clin. Oncol., vol. 31, no. 32, pp. 4085-4091, Nov. 2013, doi:

10.1200/JCO.2013.49.6968.

[40] F. S. Lieberman et al., "Phase 2 Study of Radiation Therapy Plus Low-Dose Temozolomide Followed by Temozolomide and Irinotecan for Glioblastoma: NRG Oncology RTOG Trial 0420," Int. J. Radiat. Oncol. Biol. Phys., vol. 103, no. 4, pp. 878-886, 15 2019, doi: 10.1016/j.jijrobp.2018.11.008.

[41] D. T. Blumenthal et al., "Is more better? The impact of extended adjuvant temozolomide in newly diagnosed glioblastoma: a secondary analysis of EORTC and NRG Oncology/RTOG," Neuro-oncology, vol. 19, no. 8, pp. 1119-1126, Aug. 2017, doi: 10.1093/neuonc/nox025.

[42] M. R. Gilbert et al., "A randomized trial of bevacizumab for newly diagnosed glioblastoma," $N$. Engl. J. Med., vol. 370, no. 8, pp. 699-708, Feb. 2014, doi: 10.1056/NEJMoa1308573. 
[43] O. L. Chinot et al., "Bevacizumab plus radiotherapy-temozolomide for newly diagnosed glioblastoma," N. Engl. J. Med., vol. 370, no. 8, pp. 709-722, Feb. 2014, doi: 10.1056/NEJMoa1308345.

[44] R. Stupp et al., "Cilengitide combined with standard treatment for patients with newly diagnosed glioblastoma with methylated MGMT promoter (CENTRIC EORTC 26071-22072 study): a multicentre, randomised, open-label, phase 3 trial," Lancet Oncol., vol. 15, no. 10, pp. 1100-1108, Sep. 2014, doi: 10.1016/S1470-2045(14)70379-1.

[45] M. Weller et al., "Rindopepimut with temozolomide for patients with newly diagnosed, EGFRvIIIexpressing glioblastoma (ACT IV): a randomised, double-blind, international phase 3 trial," Lancet Oncol., vol. 18, no. 10, pp. 1373-1385, 2017, doi: 10.1016/S1470-2045(17)30517-X.

[46] M. Lim, Y. Xia, C. Bettegowda, and M. Weller, "Current state of immunotherapy for glioblastoma," Nat Rev Clin Oncol, vol. 15, no. 7, pp. 422-442, 2018, doi: 10.1038/s41571-018-0003-5.

[47] R. Stupp et al., "Effect of Tumor-Treating Fields Plus Maintenance Temozolomide vs Maintenance Temozolomide Alone on Survival in Patients With Glioblastoma: A Randomized Clinical Trial," JAMA, vol. 318, no. 23, pp. 2306-2316, 19 2017, doi: 10.1001/jama.2017.18718.

[48] W. Wick et al., "Temozolomide chemotherapy alone versus radiotherapy alone for malignant astrocytoma in the elderly: the NOA-08 randomised, phase 3 trial," Lancet Oncol., vol. 13, no. 7, pp. 707715, Jul. 2012, doi: 10.1016/S1470-2045(12)70164-X.

[49] J. Gállego Pérez-Larraya et al., "Temozolomide in elderly patients with newly diagnosed glioblastoma and poor performance status: an ANOCEF phase II trial," J. Clin. Oncol., vol. 29, no. 22, pp. 3050-3055, Aug. 2011, doi: 10.1200/JC0.2011.34.8086.

\section{Tables}

Table 1: Patient and treatment characteristics. Patients with several pre-radiochemotherapy (RCT) neurological disabilities were counted twice. (IM) RT = (intensity-modulated) radiotherapy; TMZ = temozolomide. 
Gender

Male

$73(57 \%)$

Female

55 (43\%)

Age (years)

Median [range]

74 [70-88]

$70-75.5$

73 (57\%)

$\geq 75.5$

55 (43\%)

Performance Status

0

1

2

3

Type of surgery

Complete resection

Partial resection

Biopsy

RPA class

I

II

III

IV
49 (38.5\%)

$71(55.5 \%)$

$7(5.5 \%)$

$1(1 \%)$

$64(50 \%)$

$17(13.5 \%)$

$47(36.5 \%)$

$50(39 \%)$

$30(23.5 \%)$

$43(33.5 \%)$

$5(4 \%)$

Median plasma protein concentration (g/l) [range] 66.5 [51-77]

Pre-RCT neurological disabilities

$76(59.5 \%)$

Mild motor disabilities

$26(20.5 \%)$

Severe motor disabilities

$6(4.5 \%)$

Visual disabilities $†$

$17(13.5 \%)$

Instabilities $\ddagger$

$6(4.5 \%)$

Cognitive disabilities 㧊

$10(8 \%)$

Communication disorders

$28(22 \%)$

Treatment characteristics 


\begin{tabular}{ll} 
Number of RT fractions (median, range) & $30[5-30]$ \\
IMRT & $1(1 \%)$ \\
Concomitant TMZ cycles (median, range) & $6[1-6]$ \\
Maintenance TMZ cycles (median, range) & $2[0-6]$ \\
Treatment at recurrence & $49(38.5 \%)$ \\
\hline
\end{tabular}

† e.g. homonymous hemianopias or anopsias.

‡ e.g. dizziness or proprioceptive disorders.

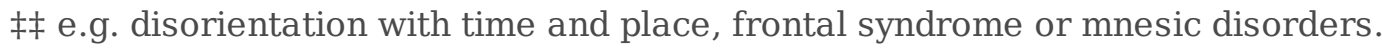

Table 2: Oncologic outcomes and rates for early ( $\leq 1$ month) radiochemotherapy (RCT)-concomitant and postRCT neurological events including, for example, the use of corticosteroids, intracranial hypertension symptoms, or hospitalization.

\begin{tabular}{ll}
\hline \multicolumn{2}{l}{$(\mathbf{n}=128)$} \\
RCT-concomitant neurological events & $50(39 \%)$ \\
Post-RCT neurological events & $58(45.5 \%)$ \\
Relapse-free survival & 9.4 (CI95\%: 8.9-10.4) \\
Median (months) & $33.3 \%(\mathrm{CI} 95 \%: 23.8-43.1)$ \\
1-year & 11.7 (CI95\%: 9.9-13.1) \\
Overall survival & $49.2 \%(\mathrm{CI} 95 \%: 40.3-57.5)$ \\
Median (months) & $15.4 \%$ (CI95\%: 9.8-22.2) \\
1-year & $2.4 \%$ (CI95\%: 0.6-6.0) \\
2-year & \\
\hline 5-year & \\
\hline
\end{tabular}

\section{Figures}




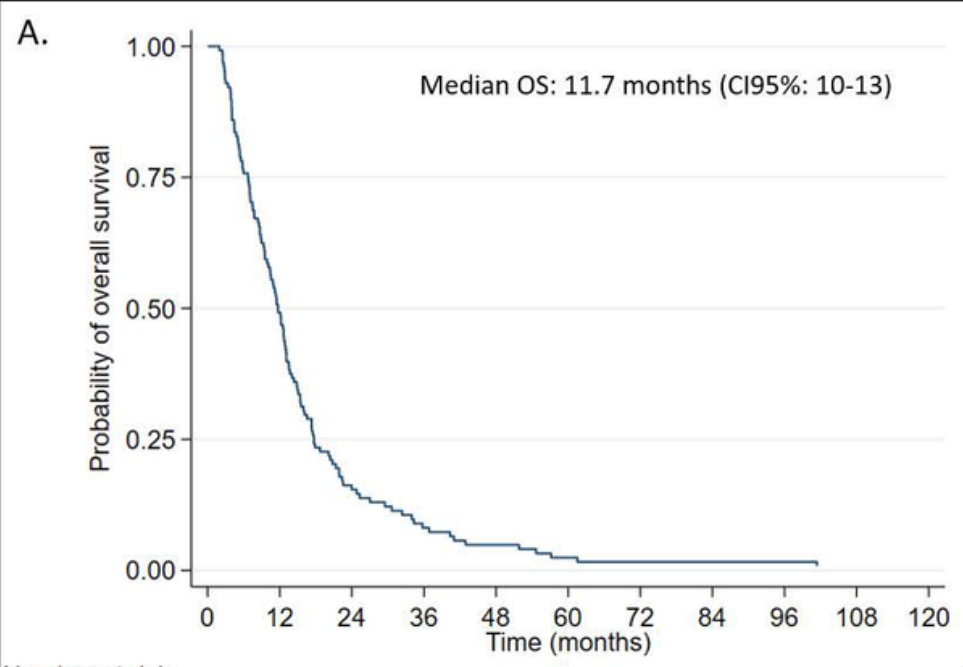

Number at risk

\section{B.}

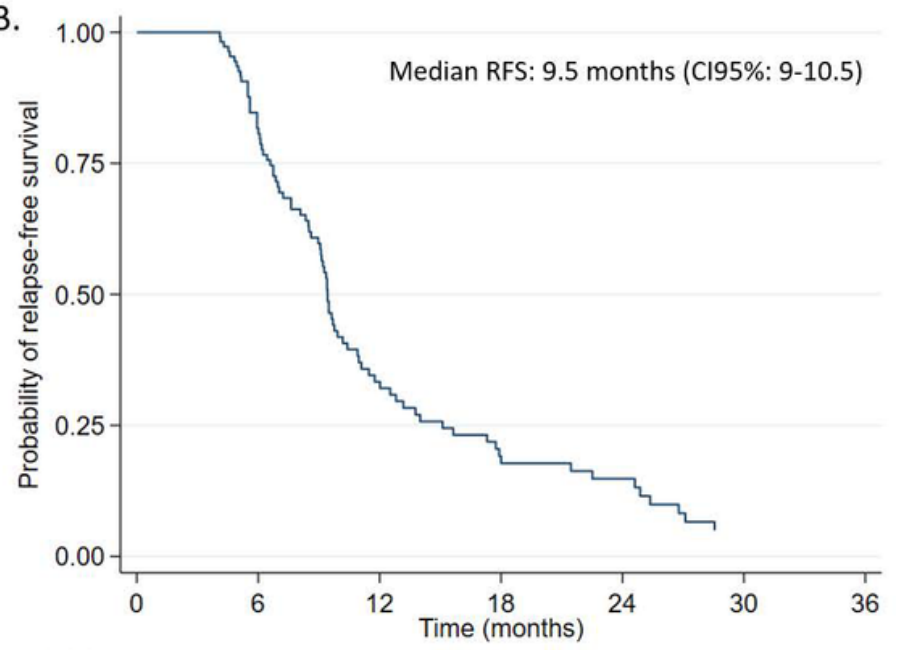

Number at risk

Figure 1

A) Overall survival (OS) and (B) relapse-free survival (RFS). Median follow-up: 1.7 months (IQR: 6.5-17.5).

A.

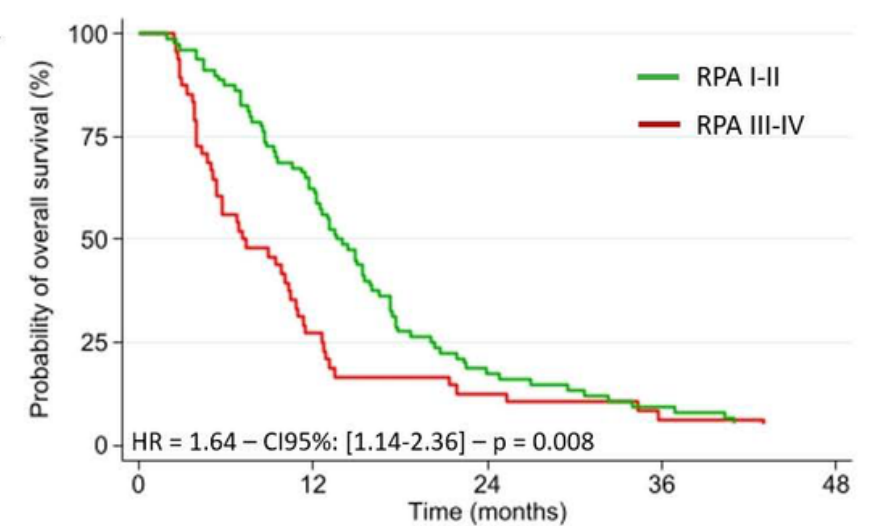

Patients at risk

RPA III-IV 48

RPA I-II 80 Overall survival Hazard Ratio and $95 \% \mathrm{CL}$

RPA (3-4 vs 1-2)
PS (1-2 vs 0)
Neurological disability
(yes vs no)
Age (ref. = 70-74)
75-79 vs ref.
80- vs ref.
Quality of surgery
PR vs CR
BR vs CR

2
4
B.

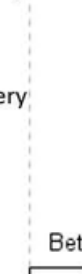

0.1

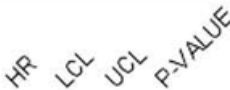

$\begin{array}{llll}1.64 & 1.14 & 2.36 & 0.008\end{array}$

$\begin{array}{llll}1.36 & 0.95 & 1.96 & 0.094\end{array}$

$\begin{array}{llll}1.04 & 0.72 & 1.49 & 0.846\end{array}$

$\begin{array}{llll}1.48 & 0.99 & 2.21 & 0.059\end{array}$

$\begin{array}{llll}1.17 & 0.7 & 1.97 & 0.541\end{array}$

$\begin{array}{llll}1.2 & 0.7 & 2.05 & 0.514\end{array}$

$\begin{array}{llll}1.66 & 1.13 & 2.44 & 0.009\end{array}$

\section{Figure 2}

(A) Overall survival (OS) depending on the RPA class and (B) OS forest plot in univariate analysis. PS = performance status; $\mathrm{C}(\mathrm{P}, \mathrm{B}) \mathrm{R}=$ complete (partial, biopsy) resection. Neurological disability = preradiochemotherapy motor, visual, instability, cognitive or communication disability. 


\section{Occurrence for early neurological events}

Odds Ratio and $95 \% \mathrm{CL}$

RPA (3-4 vs 1-2)

PS (1-2 vs 0)

Neurological disability (yes vs no)

Age (ref. $=70-74$ )

$75-79$ vs ref.

80- vs ref.

Quality of surgery

$P R$ vs $C R$

$B R$ vs $C R$

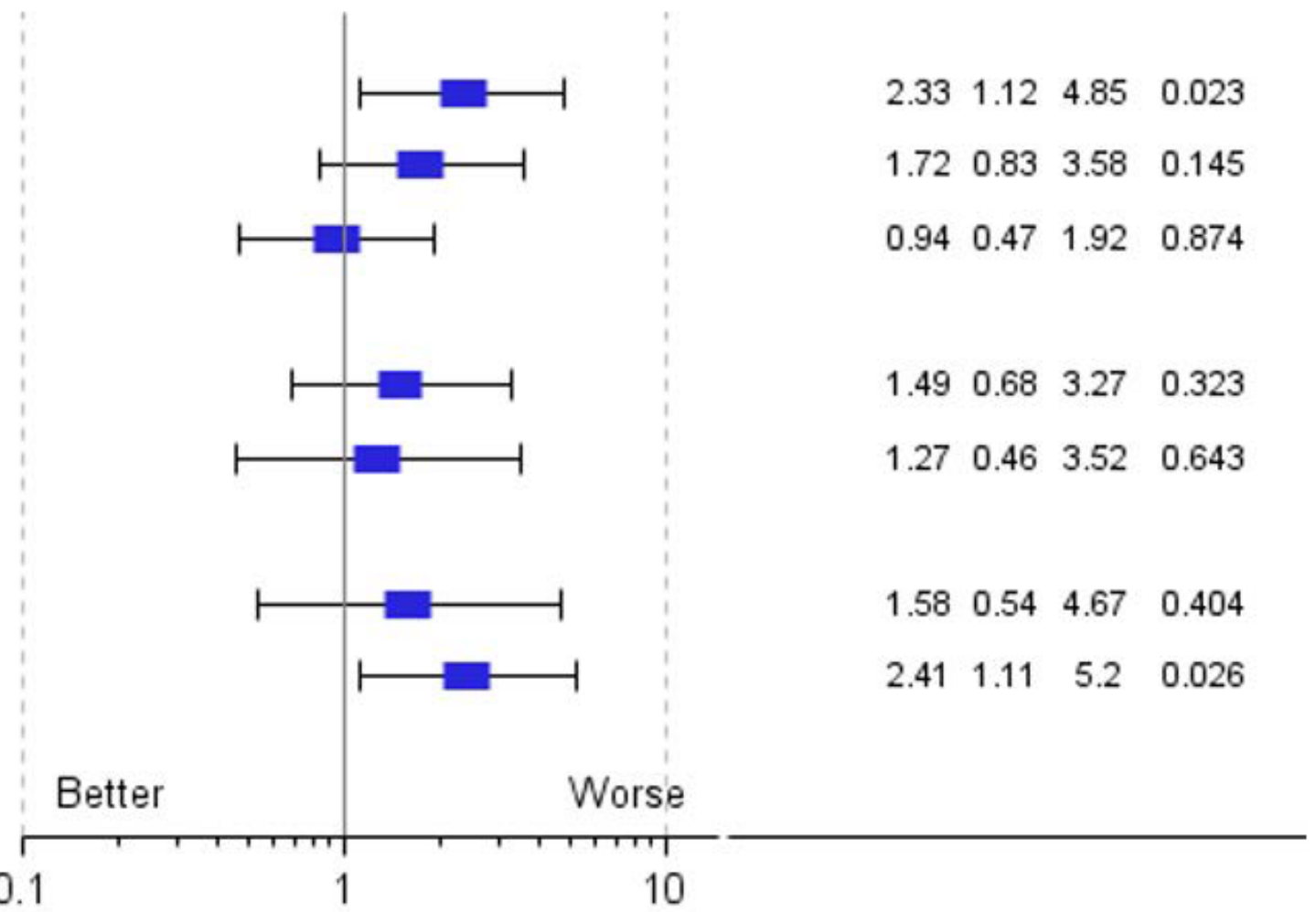

Figure 3

Forest plot in univariate analysis for the occurrence of early neurological events (defined as the occurrence of intracranial hypertension symptoms and/or use of corticosteroids and/or hospitalization before the start of temozolamide maintenance). RCT = radiochemotherapy; PS = performance status; $\mathrm{C}(\mathrm{P}, \mathrm{B}) \mathrm{R}=$ complete (partial, biopsy) resection. Neurological disability = pre-RCT motor, visual, instability, cognitive or communication disability. 